Department: Smart Home

Author:

Christina Bremer

Lancaster University

Editors:

Jeannie Albrecht (jeannie@cs.williams.edu)

A.J. Brush (ajbrush@microsoft.com)

Mike Hazas (mike.hazas@it.uu.se)

\title{
When mental models grow (c)old: A cognitive perspective on home heating automation
}

Have you ever tried to speed up a revolving door by pushing the panel in front of you, only to trigger its security mechanism and make it stop? If so, it is a simple example of a situation in which your mental representation of a system (door) and its actual functioning didn't align. In other words, you applied an incorrect mental model. Understanding mental models can be important in a variety of contexts, including smart homes, where they impact next-generation heating control.

What are mental models?

Mental models are internal representations of the physical world that allow people to understand, explain and operate a target system and predict the outcomes of their actions [1]. They are formed when people learn about their environment, usually through direct interaction. Once formed, mental models continue to evolve. Outcomes from further interaction with a target system, like the uncooperative revolving door, will be used to update previous versions in order to get closer to a workable result. Mental models can be constrained by a person's technical expertise, previous experience and cognitive abilities.

As people use target systems to achieve certain goals, the most important property of their mental models is functionality. This will be at the expense of both completeness and technical accuracy. In that sense, mental models can be seen as incomplete, yet functional, translations 
of complex real-world systems - which lead all of us to make inaccurate assumptions about how things operate on a regular basis.

From a researcher's or designer's point of view, mental models can be used to explain a person's behaviour. Adopted from the field of cognitive psychology, they have frequently been applied in ergonomics and human-computer interaction. Mental models provide a foundation to improve the design of interfaces, promote usability and encourage desired user behaviours. When a researcher reasons about a person's mental model, they will do so based on their own conceptualisation of it. That conceptualisation is, essentially, a model of a model.

\section{Why focus on home heating systems?}

Home heating systems are important from both a thermal comfort and a sustainability viewpoint. According to the Department for Business, Energy and Industrial Strategy, domestic use accounts for $29 \%$ of the UK's energy consumption, and $65 \%$ of this is for space heating [2].

What are the mental models of traditional home heating systems?

Research from the last thirty-five years has shown that it is possible to categorise people's mental models of home heating control. The resulting "shared theories" (i.e., models that are commonly held) include the feedback theory, valve theory, timer theory and switch theory [3]. In 1986, Kempton outlined the feedback theory and valve theory, after observing them in his research participants [4]. Described also as the "amateur theory", the feedback theory is a simplistic version of how a thermostat functions (for heating systems based on radiators or forced air; the other three theories are inaccurate in that context). Holders of the feedback theory believe that the system senses the room temperature, checks the thermostat set point and adjusts the boiler operation period accordingly. While not necessarily more energy efficient, the feedback theory tends to prevent users from wasting time and effort. With a valve theory, users believe that the thermostat dial controls the rate of heat flow. They might consider it their own responsibility to ensure a comfortable home temperature by changing the set point of their thermostats; these adjustments are expected to control the heat intensity in the furnace or radiators. An illustrative analogy was given by one of Kempton's interviewees who described the thermostat as a gas pedal that the operator can use to regulate the amount of heat in the system. Holding a valve theory typically leads to frequent adjustments of the thermostat, which can result in energy savings, particularly at night. 
Since 1986, the timer theory and switch theory have been added to the list. Holders of the timer theory tend to select greater set point values when they want the boiler to operate for long periods of time, whereas holders of the switch theory regard the thermostat merely as an on/off switch. Both theories are based on the assumption that it is the responsibility of the user and not the system to maintain an acceptable home temperature.

\section{What are automated home heating systems?}

In recent years, the smart and automated cousins of traditional home heating systems have become increasingly popular. Some examples are Hive, Nest, Netamo, Honeywell Evohome and Tado. Connected to the Internet and fitted with increasingly advanced algorithms, their installation comes with the promise of lower energy bills, more control for users and improved thermal comfort. Over time, the systems are designed to learn users' routines and preferences and adjust temperature settings accordingly. They frequently allow for remote control through an app or website, so that users can interact with their heating system while they are not physically in their home. Other possible functions include zoning (the home is divided into zones, which are heated individually), proximity detection (to detect when someone is nearby) and voice control.

How do people interact with automated home heating systems?

In 2011, Peffer et al. [5] reviewed the development and context of home thermostat technologies. They focused on programmable thermostats that can be used to set room temperatures for different times of the day. While programmable thermostats are not necessarily smart, Peffer et al.'s review captures some key findings about users' interaction with advanced thermostats. What they found is that the thermostats themselves are changing drastically. And as their capabilities and number of features increase, so does their complexity. Maybe it should not come as a surprise, then, that several of the reviewed studies indicate that users find the new, programmable thermostats difficult to understand. With little confidence or motivation to overcome these difficulties, almost half of them didn't use the programming features of their programmable thermostats. Misconceptions about energy and thermostats as well as cumbersome operating manuals made the situation worse. Looking at the households' energy consumption, the reviewed studies differed as to whether programmable thermostats led to significant energy savings. While some studies reported a positive effect, others did not. In several cases, households with programmable thermostats consumed more energy than those with traditional ones. Householders' attitudes towards and interactions with 
programmable thermostats also varied. While some adjusted their thermostats several times per day, others preferred to not touch theirs at all. Reasons for not interacting with their thermostats included the householders' fear of triggering unintended negative consequences and their belief that changing the thermostat settings would increase their energy consumption. In response to this variation, Peffer et al. recommended choices in the balance between user control and automation.

Looking at the next, self-learning thermostat generation, Yang and Newman (2013) conducted a study using interviews and diaries [6]. Their aim was to better understand the participants' experience of living with the Nest thermostat. While the participants welcomed the Nest overall, they did not consider it to be as helpful or intuitive as expected. This was mainly due to the system's inability to reason about user intent and the participants' difficulties in understanding how the system functions - or in other words, the inability to create a coherent mental model. Some participants developed workarounds for the perceived deficiencies, like manual revisions of automatic changes to the heating schedule. Based on these observations, Yang and Newman suggested three pillars for the design of automated technology:

- Exception flagging: Users can flag exceptional input that they do not want the system to take into account when building models and making predictions.

- Incidental intelligibility: The integration of interaction elements, like explanations about automatic features, that help the user understand the system's intelligent behaviour within the tasks they manually try to solve.

- Constrained engagement: The goal is to design technology that engages but does not overwhelm the user to keep the balance between controllability and minimal demand on user attention.

The idea of constrained engagement was taken up by Skrebe and Stumpf [7], who conducted a scenario-based study, followed by interviews. The aim of the study was to understand when users want to engage with an automated heating system and how explanations from such a system can impact user engagement. Skrebe and Stumpf found that the participants mainly interacted with the system when their thermal comfort was suboptimal or when the system did something unexpected. At the same time, the participants wanted detailed information about the system's functioning and rationale. During the study, the participants showed a tendency to continuously apply their inaccurate mental models and did not modify their approach to take account of the novel, automated system concepts. In one scenario, for example, the system switched the heating off due to high demand on the energy network. Of the fourteen participants, seven wanted to override the system by increasing the temperature, changing the heating schedule or turning the smart system off completely, going back to manual control. In 
another scenario, the system pre-heated a room ahead of schedule in order to optimise comfort for a set occupancy period. In response, nine participants tried to lower the target temperature and four tried to adapt the heating schedule.

What does this mean for the design of automated home heating systems?

Let's think back to the scenario of the automatic revolving door that you (or someone else) believed to be manual: Is it really intuitive to try to improve the user experience and behaviour by putting all our time and resources into the optimisation of the door's speed and stop algorithms? Or should we instead explain the concept of an automatic revolving door to the users before we attend to the algorithms? With smart home heating systems, the situation is similar. The studies mentioned above suggest that when we don't design home heating automation with mental models in mind, they will cause confusion and frustration. Unintended workarounds or frequent fiddling with the interface can defy the energy-saving purpose of the novel thermostats. Considering that much interaction with automated thermostats happens when there is a problem, inaccurate mental models will also make the problem-solving more difficult. We thus need mental model support in the design of automated home heating systems.

This call is supported by findings in the literature: In 2018, Revell and Stanton tested three hypotheses related to the idea that home-heating interfaces that are designed to promote appropriate mental models can help users achieve their home-heating goals [8]. During their study, twenty matched pairs of participants were asked to solve a series of tasks in a homeheating simulator using either a novel (mental model-based) or traditional interface. The results showed better task completion rates for the novel interface. Participants who used the novel interface also developed a more accurate understanding of heating controls and system elements, like sensors, sensed variables and rules of operation. The authors concluded that a mental model approach to interface design can lead to increased control over the system, which can enable users to achieve their home-heating goals.

However, beyond this promising lab-based study, one should point out the at-times-conflicting nature of automation, first described in Bainbridge's 1983 paper The Ironies of Automation [9]. Looking at home heating automation specifically, we need to acknowledge that we currently call for infrequent and minimal interaction with systems that become increasingly complex. Infrequent interaction is thereby the opposite of what would previously have led to energy saving. To deal with the increasing complexity, users require extensive explanations, which goes against the automation's underlying goal to make life easier and more convenient. The 
overarching aim is for users to be engaged, but not overwhelmed, during infrequent, problemled interactions with increasingly complex systems. Surely, this is not an easy task to accomplish.

Current tactics and design decisions have led to varying degrees of success in smart home heating systems, both from an energy saving and user experience perspective. So, if we want to increase their success, we will need to explore new paths. Approaches to interface design that carefully consider mental models can be such a path - a path that might well lead to more engagement with users and mend their continuous application of inaccurate mental models from their interaction with traditional thermostats. Considering the ironic nature of automation, embarking on this path is unlikely to magically let smart home heating realise its full potential overnight. But it can make a contribution and set the foundation for future approaches that require users to have an accurate understanding of how their heating systems work. In addition, it can help us better understand the place of agency within the automation rhetoric that "technology can do it for you". Let's consider it an idea worth warming up to - we have little to lose and much to win.

Christina Bremer is a PhD student in the Material Social Futures programme at Lancaster University. Contact her at c.bremer@lancaster.ac.uk.

[1] D. Gentner and A. L. Stevens, Mental models. Psychology Press, 2014.

[2] Department for Business, Energy and Industrial Strategy, "Energy Consumption in the UK (ECUK) 1970 to 2018," 2019. [Online]. Available: https://assets.publishing.service.gov.uk/government/uploads/system/uploads/attachment_dat a/file/820843/Energy_Consumption_in_the_UK_ECUK_MASTER_COPY.pdf. [Accessed August $10,2020]$.

[3] K. M. Revell and N. A. Stanton, "Case studies of mental models in home heat control: Searching for feedback, valve, timer and switch theories," Applied Ergonomics, vol. 45, no. 3, pp. 363-378, 2014.

[4] W. Kempton, "Two theories of home heat control," Cognitive Science, vol. 10, no. 1, pp. 7590, 1986.

[5] T. Peffer, M. Pritoni, A. Meier, C. Aragon, and D. Perry, "How people use thermostats in homes: A review," Building and Environment, vol. 46, no. 12, pp. 2529-2541, 2011. 
[6] R. Yang and M. W. Newman, "Learning from a learning thermostat: lessons for intelligent systems for the home," in Proceedings of the 2013 ACM international joint conference on Pervasive and ubiquitous computing, pp. 93-102, ACM, 2013.

[7] S. Skrebe and S. Stumpf, "An exploratory study to design constrained engagement in smart heating systems," in Proceedings of the 31st British Computer Society Human Computer Interaction Conference, pp. 1-11, BCS Learning \& Development Ltd., 2017.

[8] K. M. Revell and N. A. Stanton, "Mental model interface design: putting users in control of home heating," Building Research \& Information, vol. 46, no. 3, pp. 251-271, 2018.

[9] L. Bainbridge, "Ironies of automation," in Analysis, design and evaluation of man-machine systems, pp. 129-135, Elsevier, 1983. 